\title{
DECOMPOSITION OF RECURSIVELY ENUMERABLE DEGREES
}

\author{
A. H. LACHLAN
}

ABSTRACT. It is shown that any nonzero recursively enumerable degree can be expressed as the join of two distinct such degrees having a greatest lower bound.

Let a nonrecursive r.e. set $A$ be given. We shall show how to enumerate r.e. sets $B^{0}, B^{1}$ and $C$ such that $C$ is recursive in $A$,

$$
\begin{gathered}
B^{0} \cup B^{1}=A, \quad B^{0} \cap B^{1}=\varnothing, \\
B^{1-i} \nless_{T} B^{i} \oplus C \quad(i=0,1)
\end{gathered}
$$

and

$$
\operatorname{deg} C=\operatorname{deg}\left(B^{0} \oplus C\right) \cap \operatorname{deg}\left(B^{1} \oplus C\right) .
$$

One corollary of this result is that the so-called "nondiamond" theorem [1, Theorem 5 ] cannot be improved to read: if $b_{0}, b_{1}$ are r.e. degrees such that $b_{0} \cup b_{1}=0^{\prime}$ and $b_{0} \mid b_{1}$ then $b_{0}$ and $b_{1}$ have no greatest lower bound in the upper semilattice of r.e. degrees. The same conclusion has been reached by J. R. Shoenfield and R. I. Soare [4] who independently and at about the same time as the present author constructed r.e. degrees $b_{0}, b_{1}$ such that $b_{0} \mid b_{1}, b_{0} \cup b_{1}=0$, and $b_{0} \cap b_{1}$ exists. Our construction combines the technique for constructing minimal pairs from Lachlan [1, Theorem 1] and Yates [6] with the method of Sacks's splitting theorem [3, Theorem 1]. Our interest in this topic was awakened by Soare's article [5] where he asks whether improvement of the nondiamond theorem is possible.

We first establish some notation. Let $\left\langle\Phi_{i}: i\langle\omega\rangle\right.$ and $\left\langle\left(\Psi_{i}^{0}, \Psi_{i}^{1}\right): i\langle\omega\rangle\right.$ be standard enumerations of all partial recursive (p.r.) functionals and of all ordered pairs of p.r. functionals. We assume given an enumeration of $A$ and simultaneous uniformly effective enumerations of the p.r. functionals $\Phi_{i}, \Psi_{i}^{0}$, and $\Psi_{i}^{1}$. In describing the enumeration of $B^{0}, B^{1}$, and $C$ we often use our notations for sets and functionals to denote current approximations to them. If we wish to specify the approximation from a stage $t$ other than the current one we append " $[t]$ " to an expression. Thus $\Phi_{i}\left(B^{0}\right)[t]$ denotes the finite function obtained by applying the finite functional $\Phi_{i}[t]$, defined by the axioms of $\Phi_{i}$ enumerated before stage $t$, to $B^{0}[t]$ the set of numbers enumerated in $B^{0}$ before stage $t$. We use the same notation for a set and its characteristic function.

In the construction below we shall be satisfying the following requirements:

$N_{j}$ : If $\Psi_{j}^{0}\left(B^{0} \oplus C\right), \Psi_{j}^{1}\left(B^{1} \oplus C\right)$ are the same total function then that total function is recursive in $C$,

Received by the editors November 28, 1978 and, in revised form, September 14, 1979.

AMS (MOS) subject classifications (1970). Primary 02F30; Secondary 02F25. 


$$
R_{e, i}: B^{1-i} \neq \Phi_{e}\left(B^{i} \oplus C\right) .
$$

The lexicographic ordering of $\omega \times 2$ is denoted by $<$ and the requirement $R_{e, i}$ will be given priority according to the position of its index $(e, i)$ in this ordering.

For unary partial functions $F, G$ we let

$$
l(F, G)=\sup \{n:(\forall i<n)(F(i), G(i) \text { are both defined and equal })\}
$$

and at any stage we set

$$
l(i)=l\left(\Psi_{i}^{0}\left(B^{0} \oplus C\right), \Psi_{i}^{1}\left(B^{1} \oplus C\right)\right), \quad l_{e}^{i}=l\left(B^{1-i}, \Phi_{e}\left(B^{i} \oplus C\right)\right),
$$

and $k_{e}^{i}$ equal the least number preserving $\Phi_{e}\left(B^{i} \oplus C\right)$ on $l_{e}^{i}$. We are free to assume that $k_{e}^{i}[s+1] \geqslant k_{e}^{i}[s]$ whenever $l_{e}^{i}[s+1] \geqslant l_{e}^{i}[s]$. This will be convenient in the proof of (5) below. For each stage $s$ we define $\beta \in{ }^{\omega} 2$ by induction:

$$
\beta(n)=0 \leftrightarrow(\forall t<s)[(\beta \uparrow n)[t]=\beta \uparrow n \rightarrow l(n)[t]<l(n)] .
$$

The sequence $\beta$ is used in the satisfaction of the requirements $N_{j}$. The technique for handling these requirements is similar to that employed in the version of the minimal pair construction presented in [2]. The idea is as follows. Let $<$ denote the lexicographic ordering of ${ }^{<\omega} 2$. Fix $j$ and without loss suppose that $\Psi_{j}^{0}\left(B^{0} \oplus C\right)$ and $\Psi_{j}^{1}\left(B^{1} \oplus C\right)$ are the same total function. Let $\gamma$ be least in ${ }^{(j+1)} 2$ such that $\gamma \subset \beta[s]$ for infinitely many $s$. Note that $\gamma(j)=0$. The crucial stages for the satisfaction of $N_{j}$ are those at which $\gamma \subset \beta$. Call them $\gamma$-stages. It does not matter that $\gamma$ is unknown in the course of the construction, because we pursue the appropriate strategy for each possible value of $\gamma$ giving priority to lesser values. We ignore the Sacks requirements $R_{e, i}$ with $e \leqslant j$, which are those accorded higher priority than $N_{j}$ because they will only affect a finite number of stages. Likewise we ignore the finite number of stages with $\beta<\gamma$. The key apparatus of our construction is a strictly increasing sequence of markers $K(0), K(1), \ldots$, an initial segment of which are defined in any particular stage, and each of which is eventually fixed. With each marker $K(m)$ will be associated $F(m)$ a number not yet in $C$ which is defined when $K(m)$ is and which becomes fixed with $K(m)$. For each $m, K(m)$ will eventually be reset in a $\gamma$-stage and will be reset for the last time in a $\gamma$-stage. Let $t$ be a $\gamma$-stage in which $K(m)$ is reset and let

$$
E=\left(\Psi_{j}^{0}\left(B^{0} \oplus C\right) \uparrow l(j)\right)[t]=\left(\Psi_{j}^{1}\left(B^{1} \oplus C\right) \uparrow l(j)\right)[t]
$$

At stage $t$ we implicitly make a commitment to ensure that for all $s>t$ either

$$
E \subset\left(\Psi_{j}^{0}\left(B^{0} \oplus C\right) \uparrow l(j)[t]\right)[s]
$$

or

$$
E \subset\left(\Psi_{j}^{1}\left(B^{1} \oplus C\right) \uparrow l(j)[t]\right)[s]
$$

or

$$
(\exists i<m)(F(i)[t] \in C[s]-C[t]) .
$$

This allows us to argue at the end of the construction that $N_{j}$ will be satisfied.

The construction. We require auxiliary partial functions $K, I, E, L, F$ and $\alpha$. Initially, all these functions have empty domain and at the end of each stage they 
have as common domain some $n \in \omega$ and values in $\omega$ except for $\alpha$ whose values lie in ${ }^{<\omega} 2$. The meanings of these functions are as follows. For each $m \in \operatorname{dom} K$ we aim to attack the requirement with index $(E(m), I(m))$ by preserving $B^{I(m)}$ and $C$ on $K(m)$. When $K(m)$ is set, $L(m)$ is the length of agreement of $B^{1-I(m)}$ and $\Phi_{E(m)}\left(B^{I(m)} \oplus C\right)$, and $\alpha(m)$ is $B \uparrow E(m)$. We will choose $K(m)$ large enough to preserve $\Psi_{j}^{I(m)}\left(B^{I(m)} \oplus C\right)$ on $l(j)$ for each $j<E(m)$ such that $\beta(j)=0$ thereby helping to meet requirements $N_{j}$. Finally, $F(m)$ is a number to be enumerated in $C$ if at some later stage a number $\geqslant K(m-1)$ and $<K(m)$ is enumerated in $A$. Enumerating $F(m)$ in $C$ assists in the satisfaction of the requirements $N_{j}$ because it permits us to make a new prediction for the values of $\Psi_{j}^{0}\left(B^{0} \oplus C\right)=\Psi_{j}^{1}\left(B^{1} \oplus C\right)$ whenever necessary. This feature is what distinguishes the satisfaction of the requirements $N_{j}$ from that of the corresponding requirements in the construction of a minimal pair where $\operatorname{deg}(C)=\mathbf{0}$.

The pair $(e, i)$ is said to require attention at stage $s$ through $m$ if one of the following three possibilities holds.

$$
\begin{gathered}
m \in \operatorname{dom} K \&(e, i)=(E(m), I(m)) \& \beta \uparrow e<\alpha(m) \uparrow e \\
m \in \operatorname{dom} K \&(e, i) \leqslant(E(m), I(m)) \& \beta \uparrow e<\alpha(m) \uparrow e \\
\& \max \left(k_{e}^{i}, l_{e}^{i}\right)>K(m-1) \\
\&\left\{(e, i)=(E(m), I(m)) \rightarrow\left(\beta \uparrow e=\alpha(m) \uparrow e \& l_{e}^{i}>L(m)\right)\right\}
\end{gathered}
$$

or

$$
m=\operatorname{dom} K \& \max \left(k_{e}^{i}, l_{e}^{i}\right)>K(m-1)
$$

By convention $K(-1)=0$.

Stage $s$ will have two parts. In part one we let $(e, i)$ be the least pair requiring attention and we let $m$ be the least number through which $(e, i)$ requires attention. Delete any values the auxiliary functions may have for arguments $>m$. Set $E(m)=e, I(m)=i$, and $\alpha(m)=\beta \uparrow e$. If (1) holds set $L(m)=L(m)[s]$ and $K(m)$ equal to the least number such that

$$
K(m) \geqslant K(m)[s] \&(\forall j<e)\left(\beta(j)=0 \rightarrow K(m) \text { preserves } \Psi_{j}^{i}\left(B^{i} \oplus C\right) \text { on } l(j)\right) .
$$

Otherwise set $L(m)=l_{e}^{i}$ and $K(m)$ equal to the least number such that

$$
\begin{aligned}
K(m) \geqslant & \max \left(k_{e}^{i}, l_{e}^{i}\right) \&(m \in \operatorname{dom} K[s] \rightarrow K(m) \geqslant K(m)[s]) \\
& \&(\forall j<e)\left(\beta(j)=0 \rightarrow K(m) \text { preserves } \Psi_{j}^{i}\left(B^{i} \oplus C\right) \text { on } l(j)\right) .
\end{aligned}
$$

In either case set $F(m)$ equal to the first unused number $>K(m)$. Part one is vacuous if there is no pair requiring attention.

In part two the next member, say $n$, is enumerated in $A$. If $n>\operatorname{rng} K$ enumerate $n$ in $B^{0}$. Otherwise let $m$ be the least number such that $n<K(m)$. Enumerate $n$ in $B^{1-I(m)}, F(m)$ in $C$, and then set $F(m)$ equal the first unused number $>K(m)$. Delete all values of the auxiliary functions for arguments $>m$. This completes the construction. 
We she!l now show that at the end of any stage

$$
m \in \operatorname{dom} K \rightarrow K(m)>L(m)
$$

and

$$
\begin{aligned}
m_{0}<m_{1} \in \operatorname{dom} K \rightarrow\left[K\left(m_{0}\right)<K\left(m_{1}\right)\right. \\
. \& .\left(E\left(m_{0}\right), I\left(m_{0}\right)\right)<\left(E\left(m_{1}\right), I\left(m_{1}\right)\right) \rightarrow \alpha\left(m_{0}\right) \uparrow E\left(m_{0}\right)<\alpha\left(m_{1}\right) \uparrow E\left(m_{1}\right) \\
\left.. \& .\left(E\left(m_{1}\right), I\left(m_{1}\right)\right)<\left(E\left(m_{0}\right), I\left(m_{0}\right)\right) \rightarrow \alpha\left(m_{0}\right) \uparrow E\left(m_{1}\right)<\alpha\left(m_{1}\right) \uparrow E\left(m_{1}\right)\right] .
\end{aligned}
$$

Also for stage $s$ we have

[ $m_{0}<m_{1} \in \operatorname{dom} K \&(e, i)$ receives attention through $\left.m_{0}\right]$

$$
\rightarrow\left[(e, i)<\left(E\left(m_{1}\right), I\left(m_{1}\right)\right) \& \beta \uparrow e<\alpha\left(m_{1}\right) \uparrow e\right] .
$$

The proofs of (4), (5), and (6) are by induction on the stage. We have (4) immediately from the way $K(m)$ and $L(m)$ are defined in stage $s$.

For proof by contradiction suppose that (5) fails for the first time at the end of stage $s$ through $m_{0}, m_{1} \in \operatorname{dom} K$ where $m_{0}<m_{1}$. Then $\left(E\left(m_{1}\right), I\left(m_{1}\right)\right)$ receives attention in stage $s$ through $m_{1}$ and certainly $K\left(m_{1}\right)[s+1]>K\left(m_{1}\right)[s]$ if $m_{1} \in$ $\operatorname{dom} K[s]$. If $m_{1} \notin \operatorname{dom} K[s]$ then in stage $s$ we have

$$
K\left(m_{1}\right)>\max \left(k_{e}^{i}, l_{e}^{i}\right)>K\left(m_{1}-1\right)[s]
$$

whence $K\left(m_{1}\right)[s+1]>K\left(m_{0}\right)[s+1]$. Suppose $\left(E\left(m_{0}\right), I\left(m_{0}\right)\right)<\left(E\left(m_{1}\right), I\left(m_{1}\right)\right)$ then $\alpha\left(m_{0}\right) \uparrow E\left(m_{0}\right)<\alpha\left(m_{1}\right) \uparrow E\left(m_{0}\right)$ otherwise $\left(E\left(m_{0}\right), I\left(m_{0}\right)\right)$ would require attention at stage $s$ through $m_{0}$ by (1). Suppose $\left(E\left(m_{1}\right), I\left(m_{1}\right)\right)<\left(E\left(m_{0}\right), I\left(m_{0}\right)\right)$ and $\alpha\left(m_{1}\right) \uparrow E\left(m_{1}\right)<\alpha\left(m_{0}\right) \uparrow E\left(m_{1}\right)$ then, letting $e=E\left(m_{1}\right), i=I\left(m_{1}\right)$ for the rest of the argument, in stage $s$ we have

$$
\max \left(k_{e}^{i}, l_{e}^{i}\right)>K\left(m_{0}-1\right)
$$

and

$$
(e, i)=\left(E\left(m_{0}\right), I\left(m_{0}\right)\right) \cdot \rightarrow . \beta \uparrow e=\alpha\left(m_{0}\right) \uparrow e \& l_{e}^{i}>L\left(m_{0}\right) .
$$

The reason for this is that these relationships certainly hold after the first part of stage $t$ where $t$ is the greatest number $<s$ such that $K\left(m_{1}\right)[t]$ is undefined. At stages $>t$ and $<s$ any number enumerated in $B^{0} \cup B^{1}$ is $>K\left(m_{1}-1\right)$ whence $k_{e}^{i}[s]<k_{e}^{i}[t] \vee l_{e}^{i}[s]<l_{e}^{i}[t] . \rightarrow . l_{e}^{i}[t]>K\left(m_{1}-1\right)[t] \& l_{e}^{i}[s]>K\left(m_{1}-1\right)[s]$. Since $K\left(m_{1}-1\right)>K\left(m_{0}\right)$ and $K\left(m_{0}\right)>L\left(m_{0}\right), l_{e}^{i}[s]>K\left(m_{1}-1\right)[s]$ implies (7) and (8). This confirms the claim that (7) and (8) hold in stage $s$. Therefore, $(e, i)$ requires attention through $m_{0}$ in stage $s$ which is the desired contradiction. This completes the proof of (5).

For (6) suppose that $m_{0}<m_{1} \in \operatorname{dom} K[s]$ and $(e, i)$ receives attention through $m_{0}$ at stage $s$. Then $(e, i)<\left(E\left(m_{0}\right), I\left(m_{0}\right)\right)$ and $\beta \uparrow e<\alpha\left(m_{0}\right) \uparrow e$ from (1) and (2). For a contradiction argument let $\left(E\left(m_{1}\right), I\left(m_{1}\right)\right)<(e, i)$. Then $m_{0}<m_{1}$ and $\left(E\left(m_{1}\right), I\left(m_{1}\right)\right)<\left(E\left(m_{0}\right), I\left(m_{0}\right)\right)$ whence by (5) $\alpha\left(m_{0}\right) \uparrow E\left(m_{1}\right)<\alpha\left(m_{1}\right) \uparrow E\left(m_{1}\right)$. Since $\beta \uparrow e<\alpha\left(m_{0}\right) \uparrow e$ and $m_{0}<m_{1}$ we have $\beta \uparrow E\left(m_{1}\right)<\alpha\left(m_{1}\right) \uparrow E\left(m_{1}\right)$. Thus 
$\left(E\left(m_{1}\right), I\left(m_{1}\right)\right)$ requires attention through $m_{1}$ at stage $s$ which contradicts $\left(E\left(m_{0}\right), I\left(m_{0}\right)\right)$ receiving attention. Therefore, $(e, i)<\left(E\left(m_{1}\right), I\left(m_{1}\right)\right)$. As noted $\beta \uparrow e<\alpha\left(m_{0}\right) \uparrow e$ and by (5) $\alpha\left(m_{0}\right) \uparrow e<\alpha\left(m_{1}\right) \uparrow e$ since $e<E\left(m_{0}\right), E\left(m_{1}\right)$. Therefore, $(e, i)<\alpha\left(m_{1}\right) \uparrow e$ which completes the proof.

Correctness of the construction. We first show that in the limit each of the auxiliary functions is total. For proof by contradiction let $\boldsymbol{m}$ be the least number such that either $K(m)$ is eventually never defined or is reset possibly to the same value infinitely often. Suppose $K(m)$ is eventually never defined then $B^{1}$ is finite whence there exists $e$ such that $B^{1}=\Phi_{e}\left(B^{0} \oplus C\right)$. Since $(e, 0)$ requires attention infinitely often and $l_{e}^{0}$ tends to infinity we have a contradiction. Thus $K(m)$ is reset infinitely often. The value of $K(m)$ is deleted at most finitely often in part two of a stage. Hence eventually $K(m)$ is always defined at the end of a stage. Once $K(m)$ is always defined $(E(m), I(m))$ and $\alpha(m)$ are nonincreasing, whence $E(m), I(m)$ and $\alpha(m)$ are eventually fixed. From that point on when $K(m)$ is reset it means that (2) holds with $e=E(m)$ and $i=I(m)$, and in particular that $l_{E(m)}^{I(m)}$ has increased since the last time $K(m)$ was set. It follows that $K(m)$ and $L(m)$ increase without bound. Since $K(m-1)$ becomes fixed, eventually any number $<K(m)$ entering $A$ is enumerated in $B^{1-I(m)}$ and no number $<K(m)$ is enumerated in $B^{I(m)}$ or $C$. In the limit $B^{I(m)}$ and $C$ are recursive, $\operatorname{deg} B^{1-I(m)}=\operatorname{deg} A$, and $\Phi_{E(m)}\left(B^{I(m)} \oplus C\right)=$ $B^{1-I(m)}$. This contradiction establishes our claim.

Suppose for contradiction that $B^{1-i}=\Phi_{e}\left(B^{i} \oplus C\right)$. Choose $\gamma \in \in^{e} 2$ such that for infinitely many $m, \alpha(m) \uparrow e=\gamma$. Fix $m$ such that $(e, i)<(E(m), I(m))$ and $\alpha(m) \uparrow e=\gamma$. This is possible because from (5) for each $f<\omega$ and $j<2$ there are $<2^{f}$ values of $n$ such that $(E(n), I(n))=(f, j)$. Now choose large $s$ such that for some $n<m, K(n)$ is set or reset in stage $s$ and such that at end of stage $s$, $(e, i)<(E(n), I(n))$ and $\alpha(n) \uparrow e=\gamma$. Thus $\beta \uparrow e[s]=\gamma$. Now $l_{e}^{i}[s]>K(m)$ since $s$ is large whence $(e, i)$ requires attention through $m$ at stage $s$. Since $(E(n), I(n))$ actually receives attention at stage $s$ we have the desired contradiction.

Notice that $C$ is recursive in $A$ because a number is enumerated in $C$ only in response to a smaller number being enumerated in $A$. Next suppose

$$
\Psi_{q}^{0}\left(B^{0} \oplus C\right)=\Psi_{q}^{1}\left(B^{1} \oplus C\right)=D
$$

where $D$ is total. To complete the proof of correctness we show that $D$ is recursive in $C$. Fix $e>q$. Let $\gamma$ be the least $\delta \in E^{e} 2$ such that $(\beta \uparrow e)[s]=\delta$ for infinitely many $s$. From (1) at the end of the construction for all $m$,

$$
E(m)>e \rightarrow(\alpha(m) \uparrow e)<\gamma
$$

and for all sufficiently large $m$ we have equality on the right. From the definition of $\beta, \gamma(q)=0$. Choose $s_{0}$ such that for all $m$ if $E(m)<e$ or $\alpha(m) \uparrow e<\gamma$ in the limit then $K(m+1)[s]$ is defined for all $s>s_{0}$. Given an oracle for $C$ we can compute $D(n)$ as follows. Seek $s$ and $m$ such that $s>s_{0},(\beta \uparrow e)[s]=\gamma, l(q)[s]>n, K(m)$ is set or reset in stage $s, E(m)>e$, and none of $F(0)[s], F(1)[s], \ldots, F(m-1)[s]$ is ever enumerated in $C$. We claim that $D(n)=\Psi_{q}^{0}\left(B^{0} \oplus C\right)(n)[s]$. Call $\left(s^{\prime}, m^{\prime}\right)$ the heir of $(s, m)$ if $s^{\prime}$ is the least stage $>s$ at which $K(p)$ is reset for some $p<m$, and $K\left(m^{\prime}\right)$ is reset at stage $s^{\prime}$. Consider the sequence $(s, m),\left(s^{\prime}, m^{\prime}\right),\left(s^{\prime \prime}, m^{\prime \prime}\right), \ldots$ where 
each term is the heir of the preceding one. There is a last member $\left(s^{(k)}, m^{(k)}\right)$ because $m \geqslant m^{\prime} \geqslant m^{\prime \prime} \geqslant \cdots$ and the auxiliary functions all converge. Let $s^{(k+1)}$ denote $\omega$. For all $j \leqslant k, K\left(m^{(j)}\right)$ is not deleted in the second part of stage $s^{(j)}$ nor at any stage $>s^{(j)}$ and $<s^{(j+1)}$ because $F(h)=F(h)[s]$ for all $h<m^{(j)}$.

Let $e^{(j)}, i^{(j)}$ denote $E\left(m^{(j)}\right)\left[s^{(j)}+1\right], I\left(m^{(j)}\right)\left[s^{(j)}+1\right]$ respectively for all $j<k$. From (6) for all $j<k$ we have

$$
\left(e^{(j)}, i^{(j)}\right) \geqslant\left(e^{(j+1)}, i^{(j+1)}\right)
$$

and

$$
\begin{aligned}
\left(\alpha\left(m^{(j)}\right) \uparrow e^{(j+1)}\right)\left[s^{(j)}+1\right] & \geqslant\left(\beta \uparrow e^{(j+1)}\right)\left[s^{(j+1)}\right] \\
& =\left(\alpha\left(m^{(j+1)}\right) \uparrow e^{(j+1)}\right)\left[s^{(j+1)}+1\right] .
\end{aligned}
$$

By choice of $s_{0}, E\left(m^{(k)}\right)\left[s^{(k)}+1\right]>e$ and $\left(\alpha\left(m^{(k)}\right) \uparrow e\right)\left[s^{(k)}+1\right]>\gamma$. Hence $e^{(j)}>e$ for all $j<k$. Also, since $(\alpha(m) \uparrow e)[s+1]=(\beta \uparrow e)[s]=\gamma$, for all $j<k$ we have

$$
\left(\alpha\left(m^{(j)}\right) \uparrow e\right)\left[s^{(j)}+1\right]=(\beta \uparrow e)\left[s^{(j)}\right]=\gamma .
$$

Define $i(j)=I\left(m^{(j)}\right)\left[s^{(j)}+1\right]$. By induction on $j$, for all $j<k: l(q)\left[s^{(j)}\right]>n$,

$$
\Psi_{q}^{0}\left(B^{0} \oplus C\right)(n)\left[s^{(j)}\right]=\Psi_{q}^{1}\left(B^{1} \oplus C\right)(n)\left[s^{(j)}\right]
$$

in stage $s_{j} K\left(m^{(j)}\right)$ is set to preserve $\Psi_{q}^{i(j)}\left(B^{i(j)} \oplus C\right)(n)$, no number $<K\left(m^{(j)}\right)$ is enumerated in $B^{i(j)}$ or $C$ at a stage $\geqslant s^{(j)}$ and $<s^{(j+1)}$, and $F(h)\left[s^{(j)}\right]=F(h)[s]$ for all $h<m^{(j)}$. It is now clear that for all $t>s$ there exists $i<2$ such that

$$
\Psi_{q}^{i}\left(B^{i} \oplus C\right)(n)[t]=\Psi_{q}^{0}\left(B^{0} \oplus C\right)(n)[s] .
$$

This completes the proof of correctness.

\section{REFERENCES}

1. A. H. Lachlan, Lower bounds for pairs of recursively enumerable degrees, Proc. London Math. Soc. 16 (1966), 537-569.

2. The priority method for the construction of recursively enumerable sets (Proc. Cambridge Summer School in Logic, 1971), Lecture Notes in Math., vol. 337, Springer-Verlag, Berlin and New York, 1973.

3. G. E. Sacks, On the degrees less than 0 , Ann. of Math. (2) 77 (1963), 211-231.

4. J. R. Schoenfield and R. I. Soare, The generalized diamond theorem (abstract), Recursive Function Theory Newsletter 19 (1978), no. 219.

5. R. I. Soare, Recursively enumerable sets and degrees, Bull. Amer. Math. Soc. 84 (1978), 1149-1181.

6. C. E. M. Yates, A minimal pair of r.e. degrees, J. Symbolic Logic 31 (1966), 159-168.

Department of Mathematics, Smon Fraser University, Burnaby, Brtish Columbia, V5A 1S6 Canada 